CASE REPORT

\title{
Recurrent Epistaxis: The First Hemorrhagic Chondro-osseous Respiratory Epithelial Adenomatoid Hamartoma (COREAH) Reported in the Literature
}

\author{
David Temmermand ${ }^{1}$, Andriy Pavlenko ${ }^{2}$, Tasos Aslanidis ${ }^{3}$
}

\begin{abstract}
Chondro-osseous respiratory epithelial adenomatoid hamartomas (COREAHs) are benign entities that are rarely present within the nasal cavity but to date have only been identified in nasal polyp-like masses. This report represents the first case published with a COREAH causing epistaxis. Although these are benign lesions which are nearly $100 \%$ curable with simple surgical excisions, they remain rare and are not commonly thought of in the differential of nasal masses or as hemorrhagic lesions. Further, the patient reported here is the oldest reported and has a unique presentation.
\end{abstract}

Keywords: Endoscopic sinus surgery, Epistaxis, Nasal mass, Nasal polyp.

Otorhinolaryngology Clinics: An International Journal (2019): 10.5005/jp-journals-10003-1326

\section{INTRODUCTION}

Chondro-osseous respiratory epithelial adenomatoid hamartomas (COREAHs) are benign entities that are rarely present within the nasal cavity. Having only been described in the pathology literature around 30 years ago, there are just 7 cases described in the literature to date. And unlike the current case, all have presented as polyplike masses causing nasal obstruction. Here we report the first case to present as a hemorrhagic lesion along the anterior septum. Classic examples of hemorrhagic lesions of the nasal septum can be hemangiomas (most commonly capillary type on septum), pyogenic granulomas, carcinoma, telangiectasias (hereditary or spontaneous), or hemorrhagic polyps. Certainly, this list is not exhaustive but has not historically included COREAHs.

Our preoperative diagnosis was suspicious for a hemangioma vs pyogenic granuloma. The patient was treated conservatively in the office with multiple rounds of cautery until ultimately requiring complete surgical excision. Fortunately, surgical excision is agreed upon as the appropriate treatment modality for all of these lesions. Recurrence in the nasal cavity remains low (only 1 case demonstrated recurrence and may be attributed to incomplete margins during initial surgery due to its difficult location). ${ }^{2}$ Because of their benign nature, no further care is needed.

\section{Case Description}

$\mathrm{Mr} A$ is 75-year-old male with a several-year history of left epistaxis and no history of allergies. Approximately 1 month prior to surgery, the patient had a $3 \mathrm{~mm}$ raised lesion in Little's area of the left septum. He was cauterized in the clinic with silver nitrate multiple times over multiple days (Fig. 1). Over the subsequent 2-3 weeks, the patient demonstrated continued episodes of heavy left sided epistaxis described as pulsatile at times. Conservative management of pressure and oxymetazoline soaked cotton balls failed to control the bleeding and ultimately required a balloon-based packing (RapidRhino). On the day of surgery, there was a non-friable, irregular, white scar-like area on the left anterior/inferior nasal septum without significant ectasia of surrounding vessels. The $3 \mathrm{~mm}$
${ }^{1}$ Department of Otolaryngology-Head and Neck Surgery, Augusta University, Augusta, Georgia, USA

${ }^{2}$ Department of Pathology, Kennedy University Hospital, Stratford, New Jersey, USA

${ }^{3}$ Department of Otolaryngology-Head and Neck Surgery, Rowan University School of Osteopathic Medicine, Blackwood, USA

Corresponding Author: David Temmermand, Department of Otolaryngology-Head and Neck Surgery, Augusta University, Augusta, Georgia, USA, Phone: +1 7067216100, e-mail: temmerda@rowan.edu

How to cite this article: Temmermand D, Pavlenko A, Aslanidis T. Recurrent Epistaxis: The First Hemorrhagic Chondro-osseous Respiratory Epithelial Adenomatoid Hamartoma (COREAH) Reported in the Literature. Int J Otorhinolaryngol Clin 2019;11(1):22-23.

Source of support: Nil

Conflict of interest: None

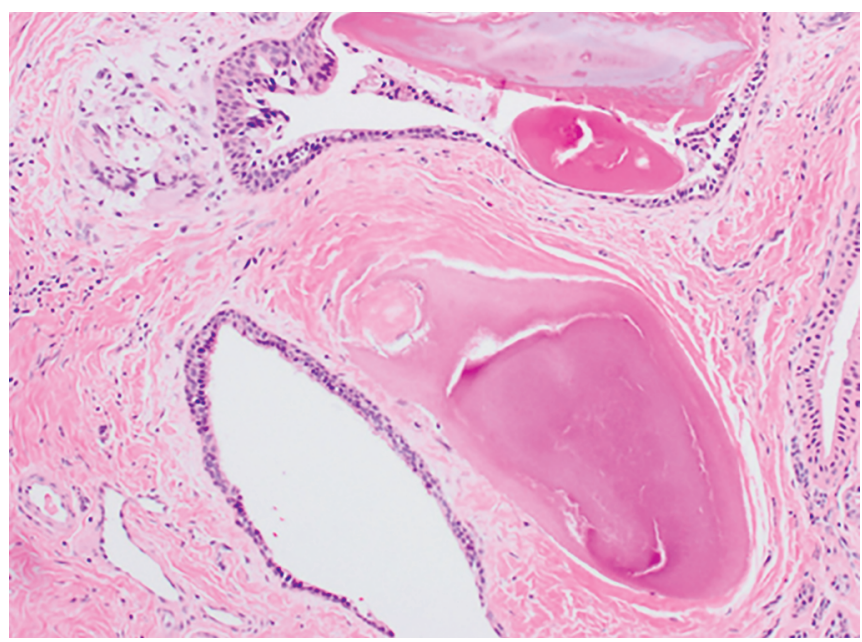

Fig. 1: Submucosal glandular proliferation with respiratory epitheliumlined mucosa, hypocellular fibromyxoid stroma, and mature bone (hematoxylin and eosin, 40x)

(c) The Author(s). 2019 Open Access This article is distributed under the terms of the Creative Commons Attribution 4.0 International License (https://creativecommons. org/licenses/by-nc/4.0/), which permits unrestricted use, distribution, and non-commercial reproduction in any medium, provided you give appropriate credit to the original author(s) and the source, provide a link to the Creative Commons license, and indicate if changes were made. The Creative Commons Public Domain Dedication waiver (http://creativecommons.org/publicdomain/zero/1.0/) applies to the data made available in this article, unless otherwise stated. 


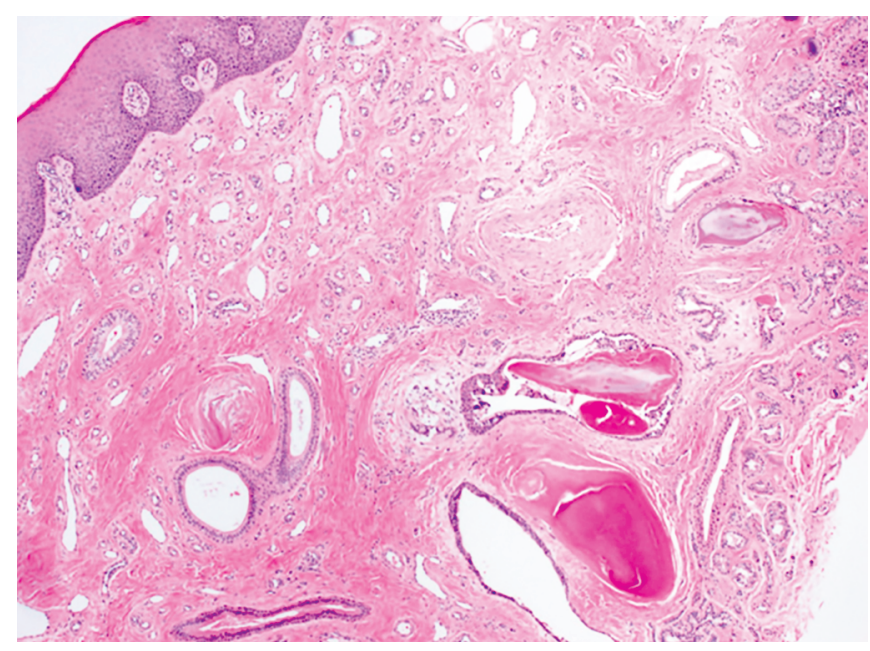

Fig. 2: Hematoxylin and eosin, 100x

area was excised down to perichondrium with the defect allowed to heal secondarily. At the 3-month follow-up, the septum has healed without incident and he has had no additional epistaxis (Fig. 2).

\section{Discussion}

Hamartomas are benign neoplasms composed of abnormal mixtures of mature cells indigenous to the local tissues with an excess of one or more cell types and can be further classified as epithelial, mesenchymal, or mixed epithelial and mesenchymal types. ${ }^{3,4}$ Within the nasal cavity, respiratory epithelial adenomatoid hamartomas (REAH) are far more common than the chondroosseous respiratory epithelial adenomatoid hamartoma (COREAH).

The etiology of COREAHs are unknown although they are histologically similar to inflammatory polyps: stromal edema, polypoid growth, increased numbers of sero-mucinous glands, vascular and fibroblastic proliferation, and mixed acute and chronic inflammatory cells. 2,3,5 Interestingly, our case was not polypoid, but given prior cautery he may have been caught at an early stage before a large polyp could have been identified.
Some also believe that REAH/COREAHs are similar to other hamartomas, which is to say that they are congenital in nature. ${ }^{3,4}$ This is unlikely to be true given the broad age spectrum this disease presents. The previously reported age range is from 3 to $68(3,7,11$, $38,34,68,59$, and now 75).

Most of the cases have been documented from the turbinates, sinuses, lateral walls, or nasal roofs. Only one was previously documented from the nasal septum but originated posteriorly. Further, all were found during cases of polyp resection secondary to nasal obstruction. ${ }^{6,7}$ This is the first case described as a nonpolypoid septal lesion and the first to present as recurrent epistaxis.

Treatment remains straightforward as simple surgical excision is enough to provide near total cure rates. ${ }^{5}$ The learning focus for this case is an expansion of the differential for hemorrhagic lesions within the nasal cavity and the ability of surgical excision to resolve this patient's epistaxis.

\section{References}

1. Iwata N, Hattori $K$, Nakagawa T, et al. Hemangioma of the nasal cavity: a clinicopathologic study. Auris Nasus Larynx 2002;29(4):335-339. DOI: 10.1016/S0385-8146(02)00028-7.

2. Fedda F, Boulos F, Sabri A. Chondro-osseous respiratory epithelial adenomatoid hamartoma of the nasal cavity. Int Arch Otorhinolaryngol 2013;17(2):218-221. DOI: 10.7162/S1809-97772013000200017.

3. Fang G, Wang C, Piao Y, et al. Chondro-osseous respiratory epithelial adenomatoid hamartoma of the nasal cavity. Pediatr Int 2016;58(3):229-231. DOI: 10.1111/ped.12777.

4. Nomura K, Oshima T, Maki A, et al. Recurrent chondro-osseous respiratory epithelial adenomatoid hamartoma of the nasal cavity in a child. Ear Nose Throat J 2014;93(1):E29-E31.

5. Choi E, Catalano PJ, Chang KG. Chondro-osseous respiratory epithelial hamartoma of the sinonasal tract. Otolaryngol Head Neck Surg 2006;134(1):168-169. DOI: 10.1016/j.otohns.2004.12.008.

6. Peric A, Vukomanovic-Durdevic B, Stanojevic I. Middle turbinate chondro-osseous respiratory epithelial adenomatoid hamartoma. B-ENT 2015;11(3):239-243.

7. Roffman E, Baredes S, Mirani N. Respiratory epithelial adenomatoid hamartomas and chondroosseous respiratory epithelial hamartomas of the sinonasal tract: a case series and literature review. Am J Rhinol 2006;20(6):586-590. DOI: 10.2500/ajr.2006.20.2947. 